\title{
Métodos não destrutivos para estimativa de densidade de área foliar em mangueira
}

\author{
Mario Zortéa Antunes Junior(1), Alessandro Ferronato(1), Susan Dignart Ferronato(1), Katiuscia Rodrigues(1), \\ Márcia Martim Pereira Gallon(1), Nídia Martineia Guerra Gomes ${ }^{(1)}$, Ana Carla Stieven ${ }^{(1)}$ \\ e José Holanda Campelo Júnior ${ }^{(1)}$
}

\begin{abstract}
(1)Universidade Federal de Mato Grosso, Avenida Fernando Corrêa, s/no, Campus Universitário, CEP 78060-900 Cuiabá, MT. E-mails: mariozortea@hotmail.com, aleferro@gmail.com, sdignard@gmail.com, kat_rodrigues@hotmail.com, gallon@cpd.umft.br, nidia@cpd.umft.br, anacarlastieven@hotmail.com, jcampelo@terra.com.br
\end{abstract}

Resumo - O objetivo deste trabalho foi estimar o número de folhas de ramos do dossel de cultivares de mangueira e estimar a densidade de área foliar utilizando, respectivamente, uma relação alométrica e um modelo de interceptação de luz. O trabalho foi conduzido com as cultivares Alfa, Roxa e Malind, na fazenda experimental da Universidade Federal de Mato Grosso, no Município de Santo Antônio do Leverger, MT. As equações testadas para a determinação do número de folhas apresentaram desempenho ótimo, com índices de confiança que variaram entre 0,85 e 0,94 , e podem ser utilizadas como alternativa para a estimativa da área foliar das três cultivares. O modelo de interceptação da luz também apresentou desempenho ótimo e bom na estimativa da densidade foliar, com índices de confiança que variaram entre 0,97 e 0,99 e 0,68 e 0,95 para as cultivares de mangueira Roxa e Malind, respectivamente.

Termos para indexação: Mangifera indica, lei de Beer-Lambert, PAR.

\section{Non-destructive methods for estimating leaf area density in mango}

\begin{abstract}
The objective of this work was to estimate the number of leaves in the branches of mango cultivars canopies and to estimate the leaf area density using, respectively, an allometric relation and a light interception model. The work was carried out with the Alfa, Roxa and Malind cultivars, grown at the experimental farm of the Universidade Federal de Mato Grosso, in the municipality of Santo Antônio do Leverger, MT, Brazil. The equations tested for determining the number of leaves had excellent performance, with confidence indexes ranging from 0,85 to 0,94 , and can be used as an alternative for estimating the leaf area of the three cultivars. The light interception model also had good performance in estimating leaf density, with confidence indexes ranging from 0,97 to 0,99 and from 0,68 to 0,95 for the Roxa and Malind mango cultivars respectively.
\end{abstract}

Index terms: Mangifera indica, Beer-Lambert law, PAR.

\section{Introdução}

Um dos elementos fundamentais para a avaliação das condições fisiológicas de plantas ou culturas é a área foliar, que depende do número e do tamanho das folhas, bem como do seu tempo de permanência na planta. A área foliar de uma cultura é amplamente utilizada como parâmetro indicativo de produtividade, pois o processo fotossintético depende da interceptação da energia luminosa e da sua conversão em energia química (Favarin et al., 2002).

Uma das variáveis usadas para descrever a área foliar é o índice de área foliar (IAF), conceituado como uma variável adimensional que representa a relação entre o total de uma das faces do tecido fotossintético e a superfície ocupada pela planta (Jonckheere et al., 2004; Monteiro et al., 2005). Outra variável importante é a densidade de área foliar (F), que consiste na área de folhas encontradas em um volume, utilizada principalmente em estudos de interceptação da luz em árvores. Essas duas variáveis são parâmetros fundamentais de entrada para os modelos de simulação de interceptação da luz, de produção de biomassa, de consumo de água, de fixação de gás carbônico e, consequentemente, para a comparação entre espécies e cultivares (Brunner, 1998; Sinoquet et al., 2007; Takeda et al., 2008).

Um dos principais problemas para a aplicação desses modelos é a dificuldade de determinação da área foliar e, por consequência, do IAF e da F, principalmente em 
espécies arbóreas. As principais dificuldades para a determinação da área foliar nestas espécies são o porte da árvore e o número de folhas de plantas adultas, o que torna essa determinação muito trabalhosa, quando não impraticável (Coelho Filho et al., 2005).

De acordo com Gower et al. (1999), a estimativa da área foliar pode ser realizada por métodos diretos e indiretos. Os diretos, com coleta periódica de amostras da planta para a medição planimétrica ou gravimétrica, são métodos destrutivos e têm como limitações a aplicação em amostras com disponibilidade limitada e a impossibilidade de avaliação, na mesma amostra, de outras características além da área foliar ao longo do tempo (Araújo et al., 2005). Os métodos indiretos, não destrutivos, usam como base medidas da transmitância da luz através das copas ou do dossel contínuo, onde normalmente se considera que as folhas têm uma distribuição aleatória nas copas. São métodos que poupam amostras e, com a utilização de equipamentos modernos, fornecem resultados rápidos $\mathrm{e}$ com razoável precisão.

Para dosséis horizontais e homogêneos, tem sido utilizada a lei de Beer-Lambert. Porém, a sua aplicação torna-se mais complexa em plantas isoladas devido à distribuição desuniforme da área foliar, que pode estar contida em um volume que tenha forma complexa.

Charles-Edwards \& Thornley (1973) propuseram um modelo de interceptação de luz em plantas isoladas por meio da aplicação da lei de Beer-Lambert, que considera as copas como formas geométricas e as trajetórias dos raios de luz através delas. Por utilizar instrumentos mais acessíveis para as medições, esse modelo pode ser uma alternativa para a obtenção de informações sobre a densidade da área foliar em espécies arbóreas.

A mangueira (Mangifera indica L.) apresenta folhagem abundante, o que resulta em sombreamento, de modo que parte da planta consome mais do que produz. Isso reduz a possibilidade de acúmulo de reservas para a produção de frutos, onera e dificulta o controle de doenças e não permite o desenvolvimento de frutos com coloração adequada para o mercado (Mouco \& Albuquerque, 2004; Kavati, 2008).

Há recomendações de poda para melhorar a disposição e o balanço da copa da árvore, o que produz uma melhora significativa na eficiência produtiva e na coloração dos frutos (Mouco \& Albuquerque, 2004). Contudo, essas recomendações não têm relação com a área foliar, de forma a preservar o equilíbrio entre a área fotossintética remanescente e a necessidade de luz e arejamento, possivelmente pelas dificuldades operacionais de estimativa da área foliar.

Este trabalho teve como objetivo estimar o número de folhas de ramos do dossel de cultivares de mangueira por meio de relação alométrica e estimar a densidade de área foliar utilizando um modelo de interceptação de luz baseado na lei de Beer-Lambert e um modelo proposto por Charles-Edwards \& Thornley (1973).

\section{Material e Métodos}

O trabalho de campo foi realizado com três cultivares de mangueira (Alfa, Roxa e Malind), cultivadas na fazenda experimental da Universidade Federal de Mato Grosso, localizada no Município de Santo Antônio do Leverger, MT $\left(15^{\circ} 47^{\prime} \mathrm{S}, 56^{\circ} 04^{\prime} \mathrm{W}\right)$, com altitude de $140 \mathrm{~m}$ acima do nível do mar. A região apresenta clima do tipo Aw pela classificação de Köppen e solos predominantemente do tipo Neossolo Litólico Distrófico (Sistema Brasileiro de Classificação de Solos, 2006).

As mangueiras foram plantadas em 2003, com espaçamento de $6 \times 8 \mathrm{~m}$, perfazendo uma área experimental total de $432 \mathrm{~m}^{2}$. Para a execução do experimento, entre agosto e dezembro de 2008 , foram monitoradas três plantas de cada cultivar, as quais foram consideradas repetições. Os tratos culturais consistiram de irrigação por microaspersão até o início do período das chuvas na região e do controle de plantas daninhas por capina mecânica.

A estimativa da área foliar (AF) foi obtida por meio de equações de regressão linear previamente calibradas com base no produto entre o comprimento e a maior largura (CL). A área foliar de 20 folhas, de tamanhos diversos, foi obtida com a utilização do programa Sigma Scan-Pro versão 5.0 (Aspire Software International, Ashburn, VA, EUA) (Jandel Scientific, 1991). Por esse procedimento, foram derivadas as seguintes equações: $\mathrm{AF}=0,7242 \times \mathrm{CL}+30,608$, com $\mathrm{R}^{2}=0,9951$; $\mathrm{AF}=0,6533 \times \mathrm{CL}+113,36$, com $\mathrm{R}^{2}=0,9638$; $\mathrm{AF}=0,6982 \times \mathrm{CL}+1,4976, \mathrm{com}^{2}=0,9992$, para as cultivares Roxa, Alfa e Malind, respectivamente.

Em cada repetição, foram medidos, quinzenalmente, de forma não destrutiva, o comprimento e a maior largura de dez folhas para a obtenção da área de uma folha média de cada cultivar. Foram medidas também as dimensões (comprimento $\mathrm{x}$ altura $\mathrm{x}$ largura) de um ramo de cada repetição. Os ramos para o monitoramento foram 
previamente definidos. A área foliar do ramo foi obtida pelo produto entre o número de folhas e a área foliar média do ramo. O volume do ramo foi calculado com a equação do volume de um elipsoide de revolução.

Foram medidas, quinzenalmente, as dimensões (comprimento $\mathrm{x}$ altura $\mathrm{x}$ largura) das copas das plantas e seus volumes foram calculados pela equação de um elipsoide de revolução. A relação entre área foliar e volume dos ramos medidos foi extrapolada para as copas, para a obtenção da área e densidade foliar total da planta.

Para a obtenção de equações alométricas que tornassem a contagem do número de folhas em um ramo mais fácil, foram realizadas regressões entre o comprimento e o número de folhas contadas em ramos de diversos tamanhos. As equações foram obtidas com a utilização do software estatístico Table Curve 2D (Systat Software Inc., San Jose, CA, EUA) (Jandel Scientific, 1991), e foram selecionadas as equações que apresentaram os melhores ajustes considerando o coeficiente de determinação $\left(R^{2}\right)$, a estatística $F$ da análise de variância, a normalidade e a homogeneidade de variâncias. Para validar as equações selecionadas, foi realizada uma amostragem em cada cultivar, apenas para essa finalidade.

Para a estimativa da densidade de área foliar (F), foi utilizado o modelo teórico de Charles-Edwards \& Thornley (1973), que estabelece que a copa ocupa um volume limitado pelo elipsoide (Equação 1):

$\left(\mathrm{x}^{2} / \mathrm{a}^{2}\right)+\left(\mathrm{y}^{2} / \mathrm{b}^{2}\right)+\left(\mathrm{z}^{2} / \mathrm{c}^{2}\right)=1$

em que $a, b$ e c são os três semieixos do elipsoide e $x, y$ e z são as coordenadas de um ponto no elipsoide. Ao se estabelecer um ponto $Q$ sobre a superfície da copa e um ponto $\mathrm{P}$ qualquer, as respectivas intensidades de luz e $\mathrm{I}_{\mathrm{q}}$ e $I_{p}$ estão relacionadas do seguinte modo (Equação 2): $\mathrm{Ip}=\mathrm{Iq} \times \exp ^{-\mathrm{KxL}}$

em que Ip é a radiação fotossinteticamente ativa (PAR - photosynthetically active radiation) global transmitida no ponto P; Iq é a PAR global incidente no ponto Q; L é o índice de área foliar ao longo da trajetória QP projetada sobre um plano normal a QP; $K$ é o coeficiente da extinção de luz, considerado como $\mathrm{K}=\mathrm{OPG} / \mathrm{sen}(\beta)$, segundo Goudriaan (1988); $\beta$ é a elevação solar (Equação 3).

$\mathrm{L}=\mathrm{F} \times \mathrm{OPG} \times \mathrm{s}$

em que $\mathrm{F}$ é a densidade de área foliar (relação área foliar por volume da copa), s é o comprimento da trajetória da luz no interior da copa e OPG é a projeção da área unitária na direção do sol; com valor de 0,5 , admite-se uma distribuição esférica ou aleatória do ângulo das folhas (Green et al., 2003). A densidade de área foliar foi estimada com a Equação 4:

$\mathrm{F}=-\ln (\mathrm{Ip} / \mathrm{Iq}) /(0,5 \times \mathrm{K} \times \mathrm{s})$

Para a estimativa de $\mathrm{F}$ pelo modelo proposto, foram efetuadas medidas da PAR, semanalmente, entre as 8 e 16h, acima e abaixo do dossel. Essas medidas foram realizadas com quatro sensores PAR-1, de fotodiodo de silício (PP Systems, Hitchin, Reino Unido), conectados a um datalogger 21X (Campbell Scientific Inc., Logan, UT, EUA). Três desses sensores foram posicionados abaixo do dossel das mangueiras e o quarto coletava os dados da PAR incidente. Também foram medidas as coordenadas das posições dos sensores em relação às plantas, no sentido das linhas e entre linhas, para posterior ajuste em relação ao norte verdadeiro (eixo $\mathrm{x}$ ), como forma de avaliar a trajetória da luz no interior da copa em função da posição do sol.

Para os cálculos, foram consideradas as medições obtidas em dias de céu limpo ou parcialmente nublado, o que resultou em quatro medições válidas para a cultivares Roxa e Malind e duas para Alfa.

No cálculo do volume, considerou-se que as copas das mangueiras têm a forma aproximada de um elipsoide de revolução e, portanto, foram efetuadas medidas da maior altura (eixo z), da maior largura na direção da linha de plantio (eixo y) e do maior comprimento na direção ortogonal à linha (eixo x).

Foi estimada a densidade foliar para os intervalos de tempo de $1,5,10,15,20,30,45$ e $60 \mathrm{~min}$. A média dos valores encontrados entre os três sensores, para cada intervalo de tempo, foi utilizada na comparação com os valores observados em cada dia de medição.

$\mathrm{Na}$ avaliação do ajuste entre valores observados e estimados para a estimativa do número de folhas, pelas equações alométricas e métodos de estimativa da densidade de área foliar, foram utilizados os seguintes indicadores: precisão, coeficiente de correlação "r"; exatidão, índice de Willmott "d" e de confiança ou desempenho "c" (Campelo Júnior, 2000). A exatidão (d) está relacionada ao afastamento dos valores estimados em relação aos observados. Esse indicador apresenta valores que variam entre zero (nenhuma exatidão) e um (exatidão perfeita). A exatidão é dada pela Equação 5:

$\mathrm{d}=1-\left\{\left[\Sigma(\mathrm{Pi}-\mathrm{Oi})^{2}\right] / \Sigma[(\mathrm{Pi}-\mathrm{O})+(\mathrm{Oi}-\mathrm{O})]^{2}\right\}$ 
em que Pi é o valor estimado, Oi é o valor observado e O é a média dos valores observados.

O índice de confiança (c), que indica o desempenho dos métodos, é representado pelo produto dos índices de precisão (r) e de exatidão (d), sendo calculado pela Equação 6:

$\mathrm{c}=\mathrm{rxd}$.

\section{Resultados e Discussão}

A relação entre o comprimento e o número de folhas do ramo apresentou um ajuste de modelo racional nas cultivares Roxa e Alfa, enquanto que, para a cultivar Malind, o modelo ajustado foi um polinômio de segundo grau. Isso indica diferenças na distribuição das folhas nos ramos entre as cultivares (Figura 1).

Mesmo com essas diferenças verificou-se, em todos os casos estudados, uma relação linear para o aumento de número de folhas até um determinado comprimento de ramo. A partir desse comprimento, foi observado um decréscimo do número de folhas, mais ou menos acentuado para cada cultivar.

Essa característica também foi observada por Ali \& Kikuzawa (2005) nos estudos de plasticidade da densidade foliar dentro da copa de Aucuba japonica Thurn., em diferentes níveis de luminosidade. Esses autores consideram que a resposta diferencial desse componente da copa depende do nível de irradiância ao qual uma planta é exposta. De forma semelhante, Teixeira \& Lima Filho (2009) encontraram uma concentração maior de folhas nas extremidades dos ramos de mangueiras, concluindo que grande parte das folhas localizadas no interior da copa recebem pouca luz e mantêm o consumo de carboidratos. Consequentemente, essas folhas atuam como drenos e não como fontes, o que resulta em redução do crescimento e da produção.

Essa característica, presente nas três cultivares estudadas, permite inferir uma distribuição desuniforme da densidade de área foliar nas copas de mangueiras, o que fere um dos principais pressupostos para a utilização da lei de Beer-Lambert nos estudos de interceptação de luz em copas isoladas de mangueira.

Outra relação alométrica para a estimativa da área foliar foi obtida por Coelho Filho et al. (2005), com um modelo exponencial da relação entre o diâmetro dos ramos e a área foliar para plantas da lima ácida 'Tahiti'. Os autores obtiveram menor precisão do ajuste com o aumento do diâmetro do ramo, considerando, no entanto, que esta abordagem poderia ser utilizada como alternativa para obter um valor médio de área foliar.

Na validação de cada modelo (Figura 2), podem ser observados valores de coeficientes de determinação $\left(\mathrm{R}^{2}\right)$ acima de $80 \%$, próximos para as três cultivares, o que indica que as equações conseguem explicar um significativo percentual da variação dos dados.

No desempenho das equações de determinação do número de folhas pela relação alométrica para manga das cultivares Roxa, Alfa e Malind, respectivamente (Tabela 1), verificou-se que o modelo proposto pode ser uma alternativa mais simples de determinação do número de folhas para a obtenção da área foliar.

Nos valores medidos pelos sensores abaixo da copa, foram observados picos na PAR, o que indica
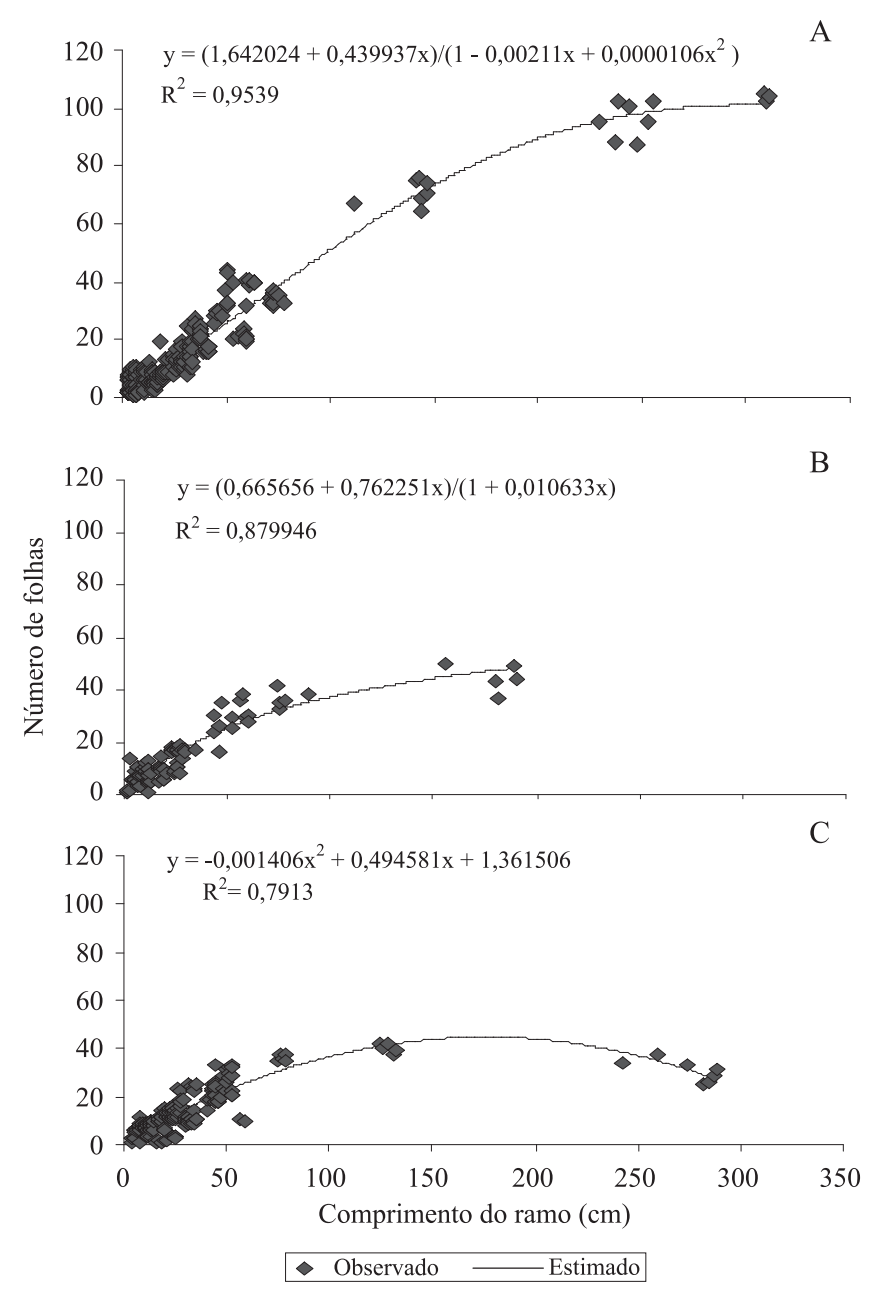

Figura 1. Equações alométricas de regressão entre o comprimento e o número de folhas de ramos de mangueira para as cultivares Roxa (A), Alfa (B) e Malind (C).

Pesq. agropec. bras., Brasília, v.44, n.12, p.1624-1630, dez. 2009 
a incidência de radiação direta, possivelmente como consequência da passagem dos raios diretamente através de espaços vazios dentro da copa (Figura 3). Observação semelhante foi feita por Campelo Júnior et al. (1996) ao quantificar a interceptação de luz em
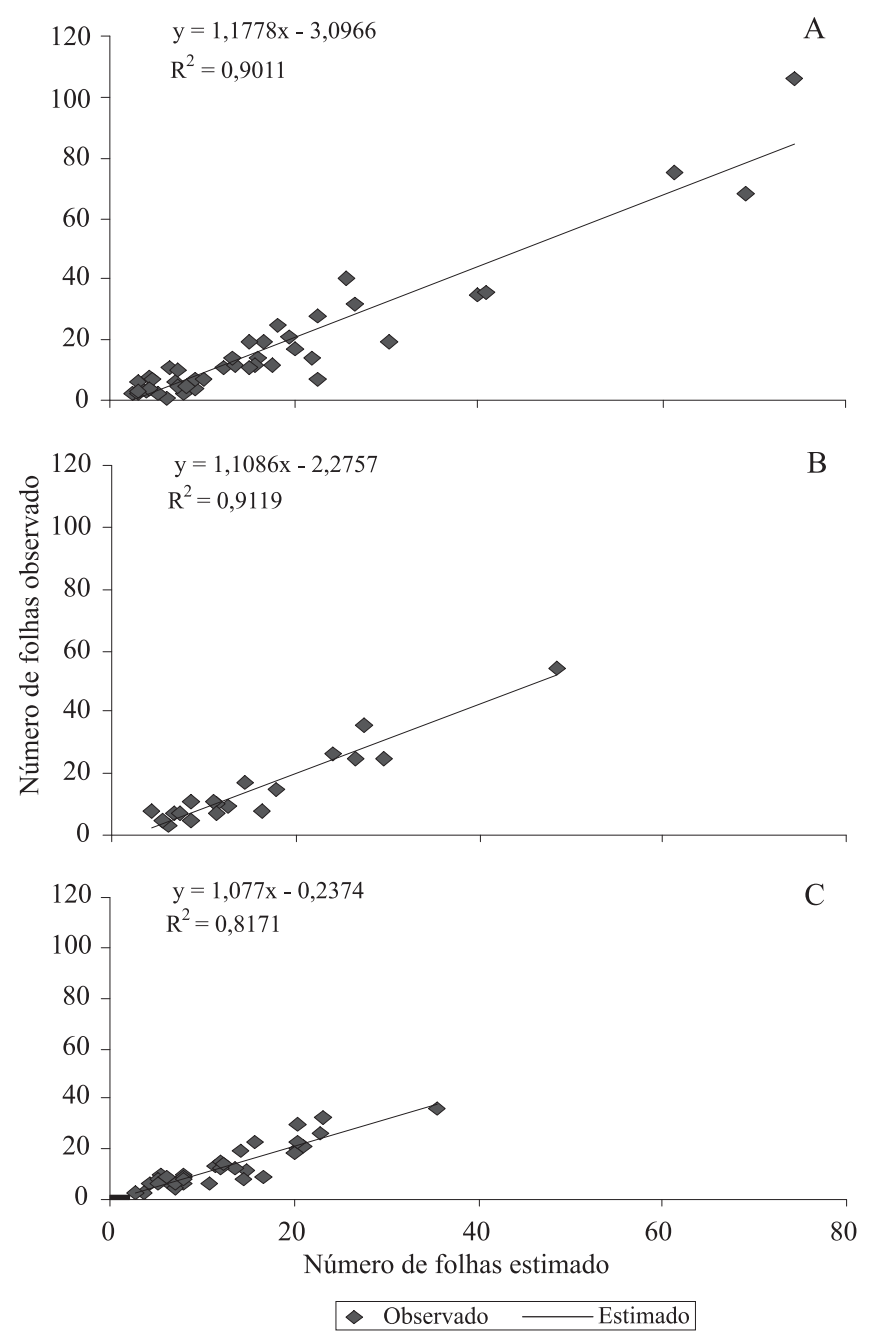

Figura 2. Relação entre valores estimados e observados encontrados na validação dos modelos para determinação, por meio de relação alométrica, do número de folhas das cultivares de manga Roxa (A), Alfa (B) e Malind (C). seringueira, também com base no modelo proposto por Charles-Edwards \& Thornley (1973).

Portanto, ainda que as mangueiras apresentem copas muito densas, a distribuição desuniforme das folhas situadas mais próximas às extremidades dos ramos permite a passagem de radiação direta, o que evita o autossombreamento excessivo.

$\mathrm{Na}$ avaliação do desempenho dos intervalos estudados de acordo com o modelo proposto por Charles-Edwards \& Thornley (1973), as equações obtidas para 20 (Roxa) e $60 \mathrm{~min}$ (Malind) apresentaram os maiores coeficientes de determinação $(0,99$ e 0,98 , respectivamente), o que indica a aproximação dos dados estimados com os observados (Figura 4).

De acordo com a classificação de Monsi et al. (1973), as cultivares de mangueiras testadas têm copas não muito densas, pois as densidades de área foliar foram menores que cinco, o que pode ser explicado pela distribuição das folhas mais próximas às extremidades dos ramos, o que confere uma aparência de copas muito densas.

Em trabalho similar, com utilização de intervalos de tempo de 15 min, Campelo Júnior et al. (1996) encontraram um $\mathrm{F}$ de $1,32 \mathrm{~m}^{2} \mathrm{~m}^{-3}$ para clones de seringueira, valor inferior aos observados neste trabalho.

A densidade de área foliar foi mais elevada para a cultivar Malind do que para a Roxa. Segundo Ali \& Kikuzawa (2005), a densidade foliar pode variar em razão de macro (densidade dos ramos que depende da razão de ramificação) e microcomponentes (comprimento do ramo, número de folhas por unidade de comprimento de ramo, tamanho das folhas), o que pode explicar a diferença da densidade foliar entre as variedades.

$\mathrm{Na}$ avaliação do desempenho do modelo proposto por Charles-Edwards \& Thornley (1973), em que foram aplicados os indicadores estatísticos utilizados por Campelo Júnior (2000), foram observados desempenhos ótimos do modelo em todos os tempos

Tabela 1. Desempenho do método de determinação do número de folhas pela relação alométrica segundo o coeficiente de correlação e os índices de Wilmott e de confiança.

\begin{tabular}{|c|c|c|c|c|}
\hline \multirow[t]{2}{*}{ Cultivar de manga } & \multirow{2}{*}{$\begin{array}{c}\text { Coeficiente de correlação "r" } \\
\text { Valor }\end{array}$} & \multirow{2}{*}{$\begin{array}{l}\text { Índice de Wilmott "d" } \\
\text { Valor }\end{array}$} & \multicolumn{2}{|c|}{ Índice de confiança "c" } \\
\hline & & & Valor & Desempenho \\
\hline Roxa & 0,9493 & 0,9959 & 0,9454 & Ótimo \\
\hline Alfa & 0,9549 & 0,9709 & 0,9271 & Ótimo \\
\hline Malind & 0,9039 & 0,9405 & 0,8501 & Ótimo \\
\hline
\end{tabular}


estudados para a variedade Roxa, com os índices de Wilmott e de confiança acima de 0,95 (Tabela 2). Esses desempenhos são indicação de que o modelo pode ser utilizado para estimar a densidade de área foliar com intervalos de tempo de 1 a 60 min sem prejuízo de precisão ou confiabilidade.



Figura 3. Radiação fotossinteticamente ativa acima (PAR incidente) e abaixo (PAR transmitida nos sensores 1, 2 e 3) do dossel sob a copa de manga cultivar Roxa, com céu limpo.
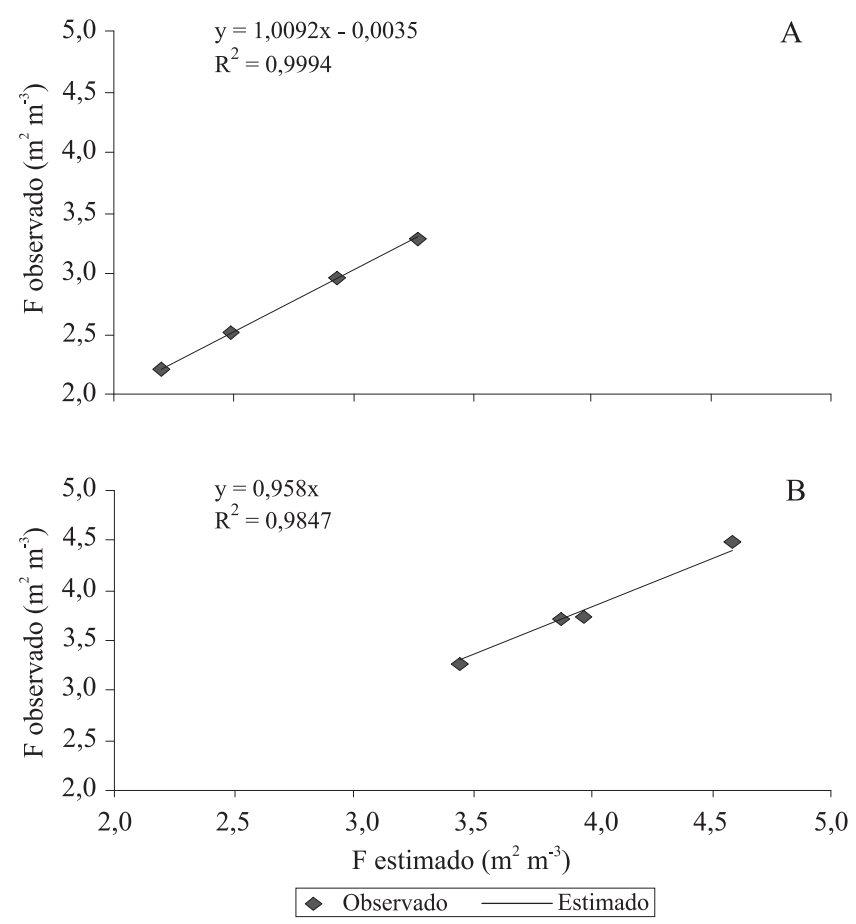

Figura 4. Relação entre a densidade de área foliar (F) observada e estimada para intervalos de tempo de 20 e 60 min para as cultivares de manga Roxa (A) e Malind (B), respectivamente.
Tabela 2. Desempenho do método de estimativa da densidade foliar de mangueira pela radiação solar interceptada pelo dossel segundo o coeficiente de correlação " $\mathrm{r}$ " e os índices de Wilmott "d" e de confiança "c".

\begin{tabular}{lcccc}
\hline $\begin{array}{l}\text { Intervalo } \\
\text { (min.) }\end{array}$ & "r" & $\begin{array}{c}\text { Índice } \\
\text { "d" }\end{array}$ & "c" & Desempenho \\
\hline \multicolumn{4}{c}{ Cultivar Roxa } \\
\hline 5 & 0,9961 & 0,9964 & 0,9925 & Ótimo \\
10 & 0,9882 & 0,9751 & 0,9636 & Ótimo \\
15 & 0,9969 & 0,9872 & 0,9841 & Ótimo \\
20 & 0,9985 & 0,9855 & 0,9840 & Ótimo \\
30 & 0,9997 & 0,9992 & 0,9989 & Ótimo \\
45 & 0,9738 & 0,9810 & 0,9553 & Ótimo \\
\hline \multicolumn{5}{c}{0,9945} \\
\hline 1 & 0,9957 & Cultivar Malind & 0,9902 & Ótimo \\
\hline 5 & 0,8306 & 0,8278 & 0,6876 & Bom \\
10 & 0,9630 & 0,9608 & 0,9252 & Ótimo \\
15 & 0,9338 & 0,9451 & 0,8825 & Ótimo \\
20 & 0,9581 & 0,9494 & 0,9096 & Ótimo \\
30 & 0,9519 & 0,9568 & 0,9108 & Ótimo \\
45 & 0,9459 & 0,9302 & 0,8799 & Ótimo \\
60 & 0,9195 & 0,9457 & 0,8696 & Ótimo \\
\hline
\end{tabular}

Para a cultivar Malind, um índice de confiança acima de 0,95 foi obtido apenas no intervalo de tempo de $60 \mathrm{~min}$, ainda que o desempenho tenha sido considerado bom para um minuto e ótimo para os demais intervalos estudados. Essa diferença de desempenho em relação à Roxa possivelmente decorre do padrão de distribuição irregular das folhas nos ramos, o que reforça a explicação de Ali \& Kikuzawa (2005) sobre a variação da densidade foliar.

Uma questão é suscitada a partir dos resultados de desempenho do modelo, que é o pressuposto da distribuição uniforme da densidade de área foliar. Mesmo que os estudos para a determinação do número de folhas por ramos demonstrem a desuniformidade na distribuição, os resultados de desempenho foram favoráveis. É importante a realização de estudos adicionais para verificar o efeito da desuniformidade na utilização do modelo para estimar a interceptação da luz.

\section{Conclusões}

1. A determinação do número de folhas por meio de relação alométrica é uma alternativa para a estimativa da área foliar das cultivares de mangueira Roxa, Alfa e Malind.

2. A densidade foliar da cultivar Roxa pode ser estimada com o modelo proposto por Charles-Edwards \& Thornley (1973) com intervalos de 1 a $60 \mathrm{~min}$.

Pesq. agropec. bras., Brasília, v.44, n.12, p.1624-1630, dez. 2009 
3. O melhor desempenho do modelo na estimativa de $\mathrm{F}$ para a cultivar Malind é obtido com o intervalo de $60 \mathrm{~min}$.

\section{Referências}

ALI, M.S.; KIKUZAWA, K. Plasticity in leaf-area density within the crown of Aucuba japonica growing under different light levels. Journal of Plant Research, v.118, p.307-316, 2005.

ARAÚJO, E.C.E.; SANTOS, E.P. dos; PRADO, C.H.B. de A. Estimativa da área foliar da mangueira (Mangifera indica L.) cvs. Tommy Atkins e Haden, utilizando dimensões lineares. Revista Brasileira de Fruticultura, v.27, p.308-309, 2005.

BRUNNER, A. A light model for spatially explicit forest stand models. Forest Ecology and Management, v.107, p.19-46, 1998.

CAMPELO JÚNIOR, J.H. Estimativa da transpiração em seringueira. Revista Brasileira de Agrometeorologia, v.8, p.35-42, 2000.

CAMPELO JÚNIOR, J.H.; MENDONÇA, E.D.D.; MENDONÇA, M.M.D.D.; PRIANTE FILHO, N.; CANEPPELE, M.A.B. Interceptação de luz pela seringueira. Revista Brasileira de Agrometeorologia, v.4, p.21-28, 1996.

CHARLES-EDWARDS, D.A.; THORNLEY, J.H.M. Light interception by an isolated plant: a simple model. Annals of Botany, v.37, p.919-28, 1973.

COELHO FILHO, M.A.; ANGELOCCI, L.R.; VASCONCELOS, M.R.B.; COELHO, E.F. Estimativa da área foliar de plantas de lima ácida 'Tahiti' usando métodos não-destrutivos. Revista Brasileira de Fruticultura, v.27, p.163-167, 2005.

FAVARIN, J.L.; DOURADO NETO, D.; GARCÍA Y GARCÍA, A.; VILLA NOVA, N.A.; FAVARIN, M. da G.G.V. Equações para a estimativa do índice de área foliar do cafeeiro. Pesquisa Agropecuária Brasileira, v.37, p.769-773, 2002.

GOUDRIAAN, J. The bare bones of leaf-angle distribution in radiation models for canopy photosynthesis and energy exchange. Agricultural and Forest Meteorology, v.43, p.155-169, 1988.

GOWER, S.T.; KUCHARIK, C.J.; NORMAN, J.M. Direct and indirect estimation of leaf area index, $f$ APAR, and net primary production of terrestrial ecosystems. Remote Sensing of Environment, v.70, p.29-51, 1999.
GREEN, S.; MCNAUGHTON, K.; WÜNSCHE, J.N.; CLOTHIER, B. Modeling light interception and transpiration of apple tree canopies. Agronomy Journal, v.95, p.1380-1387, 2003.

JANDEL SCIENTIFIC. Table Curve: curve fitting software. Corte Madera: Jandel Scientific, 1991. 280p.

JONCKHEERE, I.; FLECK, S.; NACKAERTS, K.; MUYS, B.; COPPIN, P.; WEISS, M.; BARET, F. Review of methods for in situ leaf area index determination. Part I. Theories, sensors and hemispherical photography. Agricultural and Forest Meteorology, v.121, p.19-35, 2004.

KAVATI, R. Manejo da parte aérea da mangueira. Disponível em: <http://www.nutricaodeplantas.agr.br/site/ensino/pos/Palestras William/Livromanga_pdf/09_manejodaparteaerea.pdf $>$. Acesso em: 26 out. 2008.

MONSI, M.; UCHIJIMA, Z.; OIKAWA, T. Structure of foliage canopies and photosynthesis. Annual Review of Ecology and Systematics, v.4, p.301-327, 1973.

MONTEIRO, J.E.B.A.; SENTELHAS, P.C.; CHIAVEGATO, E.J.; GUISELINI, C.; SANTIAGO, A.V.; PRELA, A. Estimação da área foliar do algodoeiro por meio de dimensões e massa das folhas. Bragantia, v.64, p.15-24, 2005.

MOUCO, M.A. do C.; ALBUQUERQUE, J.A.S. Cultivo da mangueira: sistemas de poda. Petrolina: Embrapa Semi-Árido, 2004. (Sistemas de Produção, 2).

SANTOS, H.G. dos; JACOMINE, P.K.T.; ANJOS, L.H.C. dos; OLIVEIRA, V.A. de; OLIVEIRA, J.B. de; COELHO, M.R.; LUMBRERAS, J.F.; CUNHA, T.J.F. (Ed.). Sistema brasileiro de classificação de solos. 2 ed. Rio de Janeiro: Embrapa Solos, 2006. 306p.

SINOQUET, H.; STEPHAN, J.; SONOHAT, G.; LAURI, P.É.; MONNEY, Ph. Simple equations to estimate light interception by isolated trees from canopy structure features: assessment with three-dimensional digitized apple trees. New Phytologist, v.175, p.94-106, 2007.

TAKEDA, T.; OGUMA, H.; SANO, T.; YONE, Y.; FUJINUMA, Y. Estimating the plant area density of a Japanese larch (Larix kaempferi Sarg.) plantation using a ground-based laser scanner. Agricultural and Forest Meteorology, v.148, p.428-438, 2008.

TEIXEIRA, A.H. de C.; LIMA FILHO, J.M.L. Cultivo da mangueira. Disponível em: <http://sistemasdeproducao.cnptia. embrapa.br/FontesHTML/Manga/CultivodaMangueira/clima. htm>. Acesso em: 15 abr. 2009.

Recebido em 18 de julho de 2009 e aprovado em 27 de novembro de 2009 